Journal of Organometallic Chemistry, 290 (1985) 329-339

Elsevier Sequoia S.A., Lausanne - Printed in The Netherlands

\title{
METALLORGANISCHE LEWISSÄUREN
}

XX *. REAKTIONEN VON $\left(\pi-\mathrm{C}_{5} \mathrm{H}_{5}\right)(\mathrm{CO})_{2} \mathrm{LM}-\mathrm{X}\left(\mathrm{L}=\mathrm{CO}, \mathrm{PR} \mathrm{R}_{3} ; \mathrm{M}=\mathrm{Mo}, \mathrm{W}\right.$; $\left.\mathrm{X}=\mathrm{BF}_{4}, \mathrm{PF}_{6}, \mathrm{AsF}_{6}, \mathrm{SbF}_{6}\right)$ MIT SCHWEFEL-HALTIGEN LIGANDEN

GÜNTER URBAN, KARLHEINZ SÜNKEL und WOLFGANG BECK*

Institut für Anorganische Chemie der Universität München, Meiserstr. I, 8000 München 2 (B.R.D.)

(Eingegangen den 12. Februar 1985)

\section{Summary}

The compounds $\left(\pi-\mathrm{C}_{5} \mathrm{H}_{5}\right)(\mathrm{CO})_{2} \mathrm{LM}-\mathrm{X}\left(\mathrm{L}=\mathrm{CO}, \mathrm{PR}_{3} ; \mathrm{M}=\mathrm{Mo}, \mathrm{W} ; \mathrm{X}=\mathrm{BF}_{4}\right.$, $\left.\mathrm{PF}_{6}, \mathrm{AsF}_{6}, \mathrm{SbF}_{6}\right)$ react with $\mathrm{H}_{2} \mathrm{~S}, p-\mathrm{MeC}_{6} \mathrm{H}_{4} \mathrm{SH}, \mathrm{Ph}_{2} \mathrm{~S}$ and $\mathrm{Ph}_{2} \mathrm{SO}\left(\mathrm{L}^{\prime}\right)$ to give ionic complexes $\left[\left(\pi-\mathrm{C}_{5} \mathrm{H}_{5}\right)(\mathrm{CO})_{2} \mathrm{LML}^{+} \mathrm{X}^{-}\right.$. Also sulfur-bridged complexes, [( $\pi$ $\left.\left.\mathrm{C}_{5} \mathrm{H}_{5}\right)(\mathrm{CO})_{3} \mathrm{~W}-\mathrm{SH}-\mathrm{W}(\mathrm{CO})_{3}\left(\pi-\mathrm{C}_{5} \mathrm{H}_{5}\right)\right]^{+} \mathrm{AsF}_{6}{ }^{-}$and $\left[\left(\pi-\mathrm{C}_{5} \mathrm{H}_{5}\right)(\mathrm{CO})_{3} \mathrm{M}-\mu-\right.$ $\mathrm{S}_{2} \mathrm{C}=\mathrm{NCH}_{2} \mathrm{Ph}-\mathrm{M}(\mathrm{CO})_{3}\left(\pi-\mathrm{C}_{5} \mathrm{H}_{5}\right)$, have been obtained. Reactions with $\mathrm{SO}_{2}$ and $\mathrm{CS}_{2}$ have been examined.

\section{Zusammenfassung}

Die Verbindungen $\left(\pi-\mathrm{C}_{5} \mathrm{H}_{5}\right)(\mathrm{CO})_{2} \mathrm{LM}-\mathrm{X}\left(\mathrm{L}=\mathrm{CO}, \mathrm{PR}_{3} ; \mathrm{M}=\mathrm{Mo}, \mathrm{W} ; \mathrm{X}=\mathrm{BF}_{4}\right.$, $\mathrm{PF}_{6}, \mathrm{AsF}_{6}, \mathrm{SbF}_{6}$ ) reagieren mit $\mathrm{H}_{2} \mathrm{~S}, p-\mathrm{MeC}_{6} \mathrm{H}_{4} \mathrm{SH}, \mathrm{Ph}_{2} \mathrm{~S}$ und $\mathrm{Ph}_{2} \mathrm{SO}\left(\mathrm{L}^{\prime}\right)$ zu den ionischen Komplexen $\left[\left(\pi-\mathrm{C}_{5} \mathrm{H}_{5}\right)(\mathrm{CO})_{2} \mathrm{LML}^{\prime}\right]^{+} \mathrm{X}^{-}$. Auch Schwefel-verbrückte Komplexe, $\left[\left(\pi-\mathrm{C}_{5} \mathrm{H}_{5}\right)(\mathrm{CO})_{3} \mathrm{~W}-\mathrm{SH}-\mathrm{W}(\mathrm{CO})_{3}\left(\pi-\mathrm{C}_{5} \mathrm{H}_{5}\right)\right]^{+} \mathrm{AsF}_{6}{ }^{-}$und $[(\pi-$ $\left.\left.\mathrm{C}_{5} \mathrm{H}_{5}\right)(\mathrm{CO})_{3} \mathrm{M}-\mu-\mathrm{S}_{2} \mathrm{C}=\mathrm{NCH}{ }_{2} \mathrm{Ph}-\mathrm{M}(\mathrm{CO})_{3}\left(\pi-\mathrm{C}_{5} \mathrm{H}_{5}\right)\right]$, wurden erhalten. Reaktionen mit $\mathrm{SO}_{2}$ und $\mathrm{CS}_{2}$ wurden untersucht.

\section{Einleitung}

Die schwach koordinierten Fluoroanionen in den Komplexen $\mathrm{Cp}(\mathrm{CO})_{2} \mathrm{LM}-\mathrm{X}$ $\left(\mathrm{M}=\mathrm{Mo}, \mathrm{W} ; \mathrm{L}=\mathrm{CO}, \mathrm{PR}_{3} ; \mathrm{X}=\mathrm{FBF}_{3}, \mathrm{FPF}_{5}, \mathrm{FAsF}_{5}\right.$ und $\mathrm{FSbF}_{5}$ ) lassen sich unter milden Bedingungen durch Olefine [2,3], Phosphane und Phosphite [3], Acetonitril [2] und O-Donoren wie Wasser [2,4,5], Alkohole [5], Aceton [2,5], offenkettige [5] und cyclische Ether [2,6] substituieren, wobei ionische Komplexe vom Typ $\left[\mathrm{Cp}(\mathrm{CO})_{2} \mathrm{LML}^{\prime}\right]^{+} \mathrm{X}^{-}$entstehen. Im folgenden berichten wir über Substitutionsreak-

\footnotetext{
* XIX. Mitteilung siehe Ref. 1.
} 
tionen mit Schwefelwasserstoff, Arylmerkaptanen, Diarylthioethern, Diphenylsulfoxid, Schwefeldioxid, Schwefelkohlenstoff, sowie mit $N$-Benzyldithiocarbamat.

\section{Ergebnisse}

Rührt man Lösungen von $\mathrm{CpMo}(\mathrm{CO})_{3} \mathrm{FBF}_{3}$ (1a) in $\mathrm{CH}_{2} \mathrm{Cl}_{2}$ bzw. $\mathrm{CpW}(\mathrm{CO})_{3} \mathrm{FAsF}_{5}(\mathbf{1 b})$ in $\mathrm{CHCl}_{3}$ in einer $\mathrm{H}_{2} \mathrm{~S}$-Atmosphäre bei tiefen Temperaturen, so bilden sich bald orange- bis ockerfarbene Niederschläge der SchwefelwasserstoffKomplexe 2a und 2b:

$$
\mathrm{CpM}(\mathrm{CO})_{3} \mathrm{X}+\mathrm{H}_{2} \mathrm{~S} \rightarrow\left[\mathrm{CpM}(\mathrm{CO})_{3}\left(\mathrm{H}_{2} \mathrm{~S}\right)\right]^{+} \mathrm{X}
$$

$$
(\mathbf{1 a}, \mathbf{1 b})
$$

$$
\begin{aligned}
& \text { (a: } X=\mathrm{BF}_{4}, M=M o ; \\
& \text { b: } X=\mathrm{AsF}_{6}, M=W \text { ) }
\end{aligned}
$$

Der Molybdänkomplex $2 \mathbf{a}$ ist äusserst feuchtigkeitsempfindlich und verliert schon bei $-78^{\circ} \mathrm{C}$ langsam $\mathrm{H}_{2} \mathrm{~S}$; die Wolframverbindung $2 \mathrm{~b}$ ist dagegen unter Argon und Feuchtigkeitsausschluss bei $-25^{\circ} \mathrm{C}$ mehrere Monate unzersetzt haltbar. Während in den IR-Spektren die $\nu(\mathrm{SH})$-Bande sehr intensiv erscheint, lässt sich $\mathrm{im}^{-1} \mathrm{H}-\mathrm{NMR}$ Spektrum das SH-Proton nicht eindeutig identifizieren. Lässt man $2 \mathbf{b}$ in der Kälte mit $\mathrm{Et}_{2} \mathrm{O}$ rühren, so bildet sich eine orangegelbe Lösung, die beim Einengen zur Trockene ein gelbes Pulver liefert, das durch sein IR-Spektrum als $\mathrm{CpW}(\mathrm{CO})_{3} \mathrm{SH}$ [7] erkannt wird.

Setzt man 2 Äquivalente $\mathrm{CpW}(\mathrm{CO})_{3} \mathrm{FSbF}_{5}$ (1c) in $\mathrm{CHCl}_{3}$ mit einem Äquivalent $\mathrm{NBu}_{4}{ }^{+} \mathrm{SH}^{-}$um, so entsteht der SH-verbrückte Komplex 3 (Gl. 2):

$$
\begin{aligned}
2 \mathrm{CpW}(\mathrm{CO})_{3} \mathrm{FSbF}_{5}+\mathrm{NBu}_{4}{ }^{+} \mathrm{SH}^{-} \rightarrow \\
{\left[\mathrm{Cp}(\mathrm{CO})_{3} \mathrm{~W}-\mathrm{SH}-\mathrm{W}(\mathrm{CO})_{3} \mathrm{Cp}\right]^{+} \mathrm{SbF}_{6}{ }^{-}+\mathrm{NBu}_{4}{ }^{+} \mathrm{SbF}_{6}{ }^{-} }
\end{aligned}
$$

Versetzt man eine Suspension von 3 in $\mathrm{Ft}_{2} \mathrm{O}$ mit wenig Dicyclohexylamin, so entsteht ein grüner Komplex (IR in Nujol: $\nu(C O) 2038 \mathrm{~m}, 1998 \mathrm{~s}, 1945 \mathrm{sh}, 1928 \mathrm{sh}$, $1918 \mathrm{vs}, 1895 \mathrm{vs} \mathrm{cm}^{-1}$; vermutlich $\left.\left[\mathrm{Cp}(\mathrm{CO})_{3} \mathrm{WSW}(\mathrm{CO})_{3} \mathrm{Cp}\right][8]\right)$.

Durch Reaktion von la sowie von $\left(\eta^{5}-\mathrm{C}_{9} \mathrm{H}_{7}\right) \mathrm{W}(\mathrm{CO})_{3} \mathrm{X}\left(\mathrm{X}=\mathrm{FBF}_{3}\right.$ (1d), $\mathrm{FAsF}_{5}$ (1e)) und $\mathrm{CpW}(\mathrm{CO})_{2}\left(\mathrm{P}(\mathrm{OPh})_{3}\right) \mathrm{FBF}_{3}$ (1f) mit $p-\mathrm{MeC}_{6} \mathrm{H}_{4} \mathrm{SH}$ werden die instabilen Mercaptan-Komplexe 4 erhalten (Gl. 3):

$$
\begin{aligned}
& \cdot \mathbf{a}-\mathbf{f}+p-\mathrm{MeC}_{6} \mathrm{H}_{4} \mathrm{SH} \rightarrow\left[\mathrm{Cp}^{\prime} \mathrm{M}(\mathrm{CO})_{2}(\mathrm{~L})\left(p-\mathrm{MeC}_{6} \mathrm{H}_{4} \mathrm{SH}\right)\right]^{+} \mathrm{X}^{-} \\
& (4 \mathbf{a}-\mathbf{d})
\end{aligned}
$$

Alle Mercaptan-Komplexe reagieren sehr rasch mit Spuren von Wasser, ebenso mit koordinierenden Lösungsmitteln wie Aceton unter Freisetzung des Mercaptans. Deshalb weisen alle Spektren auch die Anwesenheit von Zersetzungsprodukten auf. Der bei der Reaktion von $\mathrm{CpW}(\mathrm{CO})_{3} \mathrm{FBF}_{3}$ mit Thiophenol nach Gl. 3 in Lösung entstehende, zu 4 analoge Komplex $\left[\mathrm{CpW}(\mathrm{CO})_{3}(\mathrm{PhSH})\right]^{+} \mathrm{BF}_{4}{ }^{-}$wird mit Ether zum Thiolato-Komplex $\mathrm{CpW}(\mathrm{CO})_{3} \mathrm{SPh}$ [9] deprotoniert. Diphenylsulfid liefert mit 1a, 1c, 1e sowie $\mathrm{CpW}(\mathrm{CO})_{3} \mathrm{FBF}_{3}$ (1g) die relativ stabilen Thioether-Komplexe $\left[\mathrm{Cp}^{\prime} \mathrm{M}(\mathrm{CO})_{2}(\mathrm{~L})\left(\mathrm{Ph}_{2} \mathrm{~S}\right)\right]^{+} \mathrm{X}^{-}(5)$.

1c und $\mathrm{CpMo}(\mathrm{CO})_{3} \mathrm{FSbF}_{5}$ (1h) reagieren mit Diphenylsulfoxid zu den Verbindungen $\left[\mathrm{CpM}(\mathrm{CO})_{3}\left(\mathrm{Ph}_{2} \mathrm{SO}\right)\right]^{+} \mathrm{SbF}_{6}$ (6). 
TABELLE 1

EINGESETZTE AUSGANGSVERBINDUNGEN 1 UND PRODUKTE 2-6 $\left[\mathrm{Cp}^{\prime}(\mathrm{CO})_{2} \mathrm{LML}^{\prime}\right]^{+} \mathrm{X}^{-}$

\begin{tabular}{|c|c|c|c|c|c|}
\hline$\overline{\mathrm{Nr}}$. & $\mathrm{Cp}^{\prime}$ & $\mathbf{M}$ & $\mathbf{L}$ & $\mathrm{L}^{\prime}$ & $\mathrm{X}$ \\
\hline $1 \mathrm{a}$ & $\mathrm{C}_{5} \mathrm{H}_{5}$ & Mo & $\mathrm{CO}$ & & $-\mathrm{FBF}_{3}$ \\
\hline $\mathbf{l b}$ & $\mathrm{C}_{5} \mathrm{H}_{5}$ & w & $\mathrm{CO}$ & & FAsF $_{5}$ \\
\hline $1 \mathbf{c}$ & $\mathrm{C}_{5} \mathrm{H}_{5}$ & W & $\mathrm{CO}$ & & $\mathrm{FSbF}_{5}$ \\
\hline 1d & $\mathrm{C}_{9} \mathrm{H}_{7}$ & W & $\mathrm{CO}$ & & $\mathrm{FBF}_{3}$ \\
\hline 1e & $\mathrm{C}_{9} \mathrm{H}_{7}$ & W & $\mathrm{CO}$ & & FAsF $_{5}$ \\
\hline If & $\mathrm{C}_{5} \mathrm{H}_{5}$ & W & $\mathrm{P}(\mathrm{OPh})_{3}$ & & $\mathrm{FBF}_{3}$ \\
\hline $1 \mathrm{~g}$ & $\mathrm{C}_{5} \mathrm{H}_{5}$ & W & $\mathrm{CO}$ & & $\mathrm{FBF}_{3}$ \\
\hline 1h & $\mathrm{C}_{5} \mathrm{H}_{5}$ & Mo & $\mathrm{CO}$ & & $\mathrm{FSbF}_{5}$ \\
\hline $\mathbf{2 a}$ & $\mathrm{C}_{5} \mathrm{H}_{5}$ & Mo & $\mathrm{CO}$ & $\mathrm{H}_{2} \mathrm{~S}$ & $\mathrm{BF}_{4}$ \\
\hline 2b & $\mathrm{C}_{5} \mathrm{H}_{5}$ & w & $\mathrm{CO}$ & $\mathrm{H}_{2} \mathrm{~S}$ & $\mathrm{AsF}_{6}$ \\
\hline 3 & $\mathrm{C}_{5} \mathrm{H}_{5}$ & w & $\mathrm{CO}$ & $\left(\mathrm{C}_{5} \mathrm{H}_{5}\right)(\mathrm{CO})_{3} \mathrm{WSH}$ & $\mathrm{SbF}_{6}$ \\
\hline $4 \mathbf{a}$ & $\mathrm{C}_{5} \mathrm{H}_{5}$ & Mo & $\mathrm{CO}$ & $p-\mathrm{MeC}_{6} \mathrm{H}_{4} \mathrm{SH}$ & $\mathrm{BF}_{4}$ \\
\hline $4 \mathbf{b}$ & $\mathrm{C}_{9} \mathrm{H}_{7}$ & w & $\mathrm{CO}$ & $p-\mathrm{MeC}_{6} \mathrm{H}_{4} \mathrm{SH}$ & $\mathrm{BF}_{4}$ \\
\hline $4 c$ & $\mathrm{C}_{9} \mathrm{H}_{7}$ & w & $\mathrm{CO}$ & $p-\mathrm{MeC}_{6} \mathrm{H}_{4} \mathrm{SH}$ & $\mathrm{AsF}_{6}$ \\
\hline 4d & $\mathrm{C}_{5} \mathrm{H}_{5}$ & W & $\mathrm{P}(\mathrm{OPh})_{3}$ & $p-\mathrm{MeC}_{6} \mathrm{H}_{4} \mathrm{SH}$ & $\mathrm{BF}_{4}$ \\
\hline $\mathbf{5 a}$ & $\mathrm{C}_{5} \mathrm{H}_{5}$ & Mo & $\mathrm{CO}$ & $\mathrm{Ph}_{2} \mathrm{~S}$ & $\mathrm{BF}_{4}$ \\
\hline $\mathbf{5 b}$ & $\mathrm{C}_{5} \mathrm{H}_{5}$ & w & $\mathrm{CO}$ & $\mathrm{Ph}_{2} \mathrm{~S}$ & $\mathrm{BF}_{4}$ \\
\hline $5 c$ & $\mathrm{C}_{5} \mathrm{H}_{5}$ & W & $\mathrm{CO}$ & $\mathrm{Ph}_{2} \mathrm{~S}$ & $\mathrm{SbF}_{6}$ \\
\hline 5d & $\mathrm{C}_{9} \mathrm{H}_{7}$ & $\mathbf{w}$ & $\mathrm{CO}$ & $\mathrm{Ph}_{2} \mathrm{~S}$ & $\mathrm{AsF}_{6}$ \\
\hline 6a & $\mathrm{C}_{5} \mathrm{H}_{5}$ & Mo & $\mathrm{CO}$ & $\mathrm{Ph}_{2} \mathrm{SO}$ & $\mathrm{SbF}_{6}$ \\
\hline 6b & $\mathrm{C}_{5} \mathrm{H}_{5}$ & W & $\mathrm{CO}$ & $\mathrm{Ph}_{2} \mathrm{SO}$ & $\mathrm{SbF}_{6}$ \\
\hline
\end{tabular}

Löst man $\mathrm{CpW}(\mathrm{CO})_{3} \mathrm{FPF}_{5}$ in flüssigem $\mathrm{SO}_{2}$ auf, so erhält man eine rote Lösung, die nach Abdunsten des $\mathrm{SO}_{2}$ einen dunkelroten Feststoff (7a) liefert. Auch durch längeres Trocknen im Hochvakuum kann das violette $\mathrm{CpW}(\mathrm{CO})_{3} \mathrm{FPF}_{5}$ nicht wiedergewonnen werden. Beim Erhitzen von $7 \mathrm{a}$ im Hochvakuum werden $\mathrm{SiF}_{4}$ (vom Glas), $\mathrm{CO}$ und $\mathrm{CO}_{2}$ freigesetzt und IR-spektroskopisch nachgewiesen. Zurück bleiben $\left[\mathrm{CpW}(\mathrm{CO})_{4}\right]^{+}[2]$ und carbonylfreie Verbindungen. Auch die Reaktion von $\mathrm{CpW}(\mathrm{CO}){ }_{3} \mathrm{FSbF}_{5}$ mit gasförmigem $\mathrm{SO}_{2}$ liefert irreversibel eine purpurfarbene Verbindung, $\mathbf{7 b}$, die beim Erhitzen dieselben Gase freisetzt wie $\mathbf{7 a}$.

$7 \mathbf{a}$ und $7 \mathbf{b}$ sind extrem Wasser-empfindlich. Aus einer Suspension der Wolframverbindung in $\mathrm{Et}_{2} \mathrm{O} /$ Wasser konnte $\left\{\left[\mathrm{CpW}(\mathrm{CO})_{3}\right]_{2} \mathrm{H}\right\}^{+} \mathrm{SbF}_{6}{ }^{-}$isoliert werden.

Aus einer Lösung von $\mathrm{CpMo}(\mathrm{CO})_{3} \mathrm{FBF}_{3}$ in $\mathrm{CH}_{2} \mathrm{Cl}_{2}$ wird mit $\mathrm{CS}_{2}$ ein schwerlösliches Produkt 8a isoliert, das sich durch grosse Wasserempfindlichkeit auszeichnet. Frische Nujolverreibungen zeigen wie 7 sehr hohe Carbonylfrequenzen, die schnell an Intensität abnehmen. Daneben lassen sich sowohl IR- als auch ${ }^{1} \mathrm{H}$-NMR-spektroskopisch $\left[\mathrm{CpMO}(\mathrm{CO})_{4}\right]^{+} \mathrm{BF}_{4}{ }^{-}, \quad\left[\mathrm{CpMo}(\mathrm{CO})_{3}\left(\mathrm{H}_{2} \mathrm{O}\right)\right]^{+}$und $\left\{\left[\mathrm{CpMo}(\mathrm{CO})_{3}\right]_{2} \mathrm{H}\right\}^{+}$identifizieren [2] (siehe experimenteller Teil). Die durch Reaktion von $\mathrm{CpW}(\mathrm{CO})_{3} \mathrm{FAsF}_{5}$ mit $\mathrm{CS}_{2}$ erhaltene analoge Wolframverbindung $8 \mathrm{~b}$ ist zwar deutlich stabiler als 8a, aber immer noch sehr wasserempfindlich.

Die Tetrafluoroborato-Komplexe $\mathrm{CpM}(\mathrm{CO})_{3} \mathrm{FBF}_{3}(\mathrm{M}=\mathrm{Mo}, 1 \mathrm{1a} ; \mathrm{M}=\mathrm{W}, \mathbf{1 g})$ bilden mit $\mathrm{N}$-Benzyldithiocarbamat $\left[\mathrm{PhCH}_{2}-\mathrm{NHCS}_{2}\right]^{-}$[10], die zweikernigen Neutralverbindungen 9 (Gl. 4):

$2 \mathrm{CpM}(\mathrm{CO})_{3} \mathrm{FBF}_{3}+\mathrm{PhCH}_{2} \mathrm{NHCS}_{2}{ }^{-} \rightarrow$

$$
\begin{gathered}
{\left[\mathrm{Cp}(\mathrm{CO})_{3} \mathrm{M}-\mathrm{S}-\mathrm{C}\left(=\mathrm{NCH}_{2} \mathrm{Ph}\right)-\mathrm{S}-\mathrm{M}(\mathrm{CO})_{3} \mathrm{Cp}\right]+\mathrm{HBF}_{4}+\mathrm{BF}_{4}^{-}} \\
(\mathbf{9 a}, \mathrm{M}=\mathrm{Mo} \\
\mathbf{9 b}, \mathbf{M}=\mathbf{W})
\end{gathered}
$$


TABELLE 2

SPEKTROSKOPISCHE DATEN DER VERBINDUNGEN 2-6 UND 9

\begin{tabular}{lllll}
\hline $\mathrm{Nr}$. & $\nu(\mathrm{CO})\left(\mathrm{cm}^{-1}\right)$ & & $\delta\left(\mathrm{C}_{5} H_{5}\right)^{f}(\mathrm{ppm})$ \\
\hline 2a & $2064 \mathrm{~s}, 1960 \mathrm{vs}, \mathrm{br}^{a}$ & $2590 \mathrm{vs}$ & $(\nu(\mathrm{SH}))^{a}$ & $5.76^{c}$ \\
2b & $2063 \mathrm{~s}, 1965 \mathrm{vs}, \mathrm{br}^{a}$ & $2548 \mathrm{~s}$ & $(\nu(\mathrm{SH}))^{a}$ & $6.19^{d}$ \\
3 & $2050 \mathrm{vs}, 1980 \mathrm{sh}, 1967 \mathrm{sh}, 1952 \mathrm{vs}, 1929 \mathrm{vs}^{a}$ & $2552 \mathrm{vw}$ & $(\nu(\mathrm{SH}))^{a}$ & $6.03^{d}$ \\
4a & $2058 \mathrm{~s}, 1968 \mathrm{vs}, \mathrm{br}^{a}$ & & & \\
4b & $2050 \mathrm{~s}, 1984 \mathrm{~s}, 1964 \mathrm{~s}, 1945 \mathrm{sh}^{a}$ & & & \\
4c & $2042 \mathrm{~s}, 1944 \mathrm{vs}{ }^{b}$ & & & \\
4d & $1984 \mathrm{~s}, 1926 \mathrm{~m}, 1882 \mathrm{sh}^{a}, 1874 \mathrm{~m}^{a}$ & $2428 \mathrm{vw}$ & $(\nu(\mathrm{SH}))^{a}$ & \\
5c & $2068 \mathrm{~s}, 1995 \mathrm{~s}, 1956 \mathrm{vs}^{b}$ & & & \\
5d & $2043 \mathrm{vs}, 1971 \mathrm{~s}, 1939 \mathrm{vs}{ }^{a}$ & & & \\
6a & $2070 \mathrm{~s}, 2050 \mathrm{~m}, 2022 \mathrm{~s}, 1964 \mathrm{vs}, 1900 \mathrm{w}^{b}$ & 940 & $(\nu(\mathrm{SO}))$ & $5.85^{e}$ \\
6b & $2065 \mathrm{~s}, 2048 \mathrm{~m}, 2011 \mathrm{~m}, 1950 \mathrm{vs}, 1895 \mathrm{sh}^{b}$ & 910 & $(\nu(\mathrm{SO}))$ & $5.97^{e}$ \\
9a & $2039 \mathrm{~s}, 1968 \mathrm{~m}, 1952 \mathrm{vs}, 1941 \mathrm{vs}, 1899 \mathrm{w}, 1864 \mathrm{~s}^{a}$ & $1605 \mathrm{~m}$ & $(\nu(\mathrm{CN}))$ & \\
9b & $2037 \mathrm{~s}, 1966 \mathrm{~m}, 1943 \mathrm{vs}, 1853 \mathrm{vs}{ }^{a}$ & $1598 \mathrm{~m}$ & $(\nu(\mathrm{CN}))$ & \\
\hline
\end{tabular}

${ }^{a}$ In Nujol. ${ }^{h}$ In KBr. ${ }^{c}$ In $\mathrm{CD}_{2} \mathrm{Cl}_{2} .{ }^{d}$ In $\mathrm{CD}_{3} \mathrm{NO}_{2}$. ${ }^{e}$ In $\mathrm{CDCl}_{3} .{ }^{f}$ Für $\mathrm{C}_{9} \mathrm{II}_{7}$ werden Multipletts zwischen $\delta 6$ und $8 \mathrm{ppm}$ gefunden.

Die spektroskopischen Daten dieser roten, in polaren und unpolaren organischen Lösungsmitteln unter teilweiser Zersetzung löslichen Verbindungen sind zusammen mit denen von 2-6 in Tab. 2 zusammengefasst.

\section{Diskussion}

\section{1. $\mathrm{H}_{2} \mathrm{~S}$ - und $\mathrm{HS}^{-}$-Komplexe}

Im Unterschied zu den zahlreichen Komplexen mit $\mathrm{H}_{2} \mathrm{O}$ ist die Komplexchemie des $\mathrm{H}_{2} \mathrm{~S}$-Moleküls auf einige wenige Beispiele beschränkt: $\left[\mathrm{Ru}\left(\mathrm{NH}_{3}\right)_{5}\left(\mathrm{H}_{2} \mathrm{~S}\right)\right]^{2+}[11]$, $\left[\mathrm{W}(\mathrm{CO})_{5}\left(\mathrm{H}_{2} \mathrm{~S}\right)\right][12],\left[\mathrm{Mn}(\mathrm{CO})_{4}\left(\mathrm{PPh}_{3}\right)\left(\mathrm{H}_{2} \mathrm{~S}\right)^{+} \mathrm{BF}_{4}^{-}[13]\right.$ und $\left[\mathrm{Re}(\mathrm{CO})_{5}\left(\mathrm{H}_{2} \mathrm{~S}\right)\right]^{+}[14]$. Der Ruthenium- und Wolfram-Komplex zeichnen sich durch ihre Brönsted-Acidität gegenüber schwachen Basen aus; selbst Bromid kann $\left[\mathrm{W}(\mathrm{CO})_{5}\left(\mathrm{H}_{2} \mathrm{~S}\right)\right]$ deprotonieren. An $d^{8}$ - und $d^{10}$-Metallkomplexe lässt sich $\mathrm{H}_{2} \mathrm{~S}$ oxidativ addieren $[15,16]$. Versuche, $\mathrm{H}_{2} \mathrm{~S}$-Komplexe durch Protonierung von Hydrogensulfido-Komplexen zu erhalten, führten zu Dimerisierung mit $\mu$-SH-Brücken [17]. Beim Versuch, [CpMn(CO) ${ }_{2}\left(\mathrm{H}_{2} \mathrm{~S}\right)$ ] darzustellen, wurde statt dessen $\left[\mathrm{CpMn}(\mathrm{CO})_{2}\right]_{2}(\mu-S)$ erhalten [18]. Die Reaktion von $\mathrm{W}(\mathrm{CO})_{6} \mathrm{mit} \mathrm{SH}^{-}$führte je nach verwendetem Kation zu monomerem [W(CO) $\left.{ }_{5} \mathrm{SH}\right]^{-}$ oder dimerem $\left\{\left[(\mathrm{CO})_{5} \mathrm{~W}\right]_{2}(\mu-\mathrm{SH})\right\}^{-}$, wobei sich das Monomere durch NaOEt nicht deprotonieren lässt, während das Dimere zum Dianion $\left[(\mathrm{CO})_{5} \mathrm{~W}\right]_{2}(\mu-\mathrm{S})^{2-}$ reagiert [17]. $\mathrm{CpM}(\mathrm{CO})_{3} \mathrm{SH}(\mathrm{M}=\mathrm{Mo}, \mathrm{W})$ konnten durch Einschiebung von Schwefel in die Metall-H-Bindung erhalten werden [7]. Meist sind SH-Komplexe nur als Dimere, wie z.B. $\left[\mathrm{Mn}(\mathrm{CO})_{4} \mathrm{SH}\right]_{2}[7,19] \mathrm{zu}$ isolieren. Während die Mo-Verbindung $2 \mathrm{a} \mathrm{zu}$ instabil für weitergehende Untersuchungen ist, lassen sich $\mathbf{2 b}$ und $\mathbf{3}$ von schwachen Basen, z.B. $\mathrm{Et}_{2} \mathrm{O}$, wie die oben genannten Pentaammin- und Pentacarbonyl-Komplexe $[11,12]$ deprotonieren (Gl. 5):

$\left[\mathrm{CpW}(\mathrm{CO})_{3}(\mathrm{SHR})\right]^{+}+\mathrm{B} \rightarrow \mathrm{CpW}(\mathrm{CO})_{3} \mathrm{SR}+\mathrm{BH}^{+}$

$(\mathbf{2 b}, \mathbf{R}=\mathbf{H}$;

3, $\left.\mathrm{R}=\mathrm{W}(\mathrm{CO})_{3} \mathrm{Cp}\right)$ 
Auch der Farbwechsel bei der Deprotonierung von 3 von gelb nach grün entspricht dem bei der analogen Reaktion von $\left\{\left[(\mathrm{CO})_{5} \mathrm{~W}\right]_{2}(\mu-\mathrm{SH})\right\}^{-} \mathrm{zu}\left\{\left[(\mathrm{CO})_{5} \mathrm{~W}\right]_{2}(\mu-\mathrm{S})\right\}^{2-}$ [17]. Kürzlich konnte der grüne Komplex $\left[\mathrm{CpW}(\mathrm{CO})_{3}\right]_{2}(\mu-\mathrm{S})$ [8] erhalten werden, dessen IR-Spektrum mit dem von uns bei der Deprotonierung von 3 erhaltenen nicht übereinstimmt. Denkbar ist auch eine Decarbonylierung von $3 \mathrm{zu}$ einem, dem ebenfalls bekannten $\left[\mathrm{CpCr}(\mathrm{CO})_{2}\right]_{2}(\mu-\mathrm{S})[20]$ analogen Produkt. Es ergab sich kein Hinweis für die Bildung eines Trismetallasulfoniumkations $\left\{\left[\mathrm{CpM}(\mathrm{CO})_{2}\right]_{3} \mathrm{~S}\right\}^{+}$, das mit $\mathbf{M}=$ Mo bekannt ist [21].

\section{Mercaptan-, Thioether- und Diphenylsulfoxid-Komplexe}

Obwohl eine Vielzahl von Komplexen mit endständigen und verbrückenden Thiolat-Anionen beschrieben wurde [22], sind nur wenige Mercaptan-Komplexe bekannt. Dies liegt vor allem an der durch Koordination erhöhten Acidität des SH-Protons. So wirkt der Thiophenol-Komplex $\left[\mathrm{CpFe}(\mathrm{CO})_{2}(\mathrm{PhSH})\right]^{+}$gegenüber einer Reihe auch schwach basischer Lösungsmittel als Brönsted-Säure [23]. Ebenso wird $\left[\mathrm{CpW}(\mathrm{CO})_{3}(\mathrm{PhSH})\right]^{+}$bereits mit Ether zu $\mathrm{CpW}(\mathrm{CO})_{3} \mathrm{SPh}$ deprotoniert.

Thioether, vor allem die cyclischen und mehrzähnigen Vertreter dieser Klasse, besitzen eine umfangreiche Komplexchemie [24]. Als Beispiele seien hier nur $\left[\left(\mathrm{C}_{5} \mathrm{Me}_{5}\right) \mathrm{Rh}\left(\mathrm{SMe}_{2}\right)_{3}\right]^{2+}[25], \quad\left[\mathrm{CpFe}(\mathrm{CO})_{2}(\mathrm{PhSMe})\right]^{+}$[26] und [CpCo( $\left.\mathrm{PMe}_{3}\right)-$ $(\mathrm{L})(\mathrm{PhSMe})]^{2+}[27]$ und das instabile $\left[\mathrm{CpMn}(\mathrm{CO})_{2}\left(\mathrm{Ph}_{2} \mathrm{~S}\right)\right]$ [28] genannt. Auch in 5 lässt sich der Thioether durch Donorsolventien oder Bromid (KBr-Pressling!) leicht verdrängen.

Bei Sulfoxid-Komplexen [29] kann Metall-O- oder Metall-S-Koordination aus den IR-aktiven $\nu$ (SO)-Banden abgeleitet werden. Bei S-Bindung erscheint $\nu$ (SO) oberhalb $1090 \mathrm{~cm}^{-1}$, bei O-Bindung unterhalb $950 \mathrm{~cm}^{-1}$ [30]. So wird für $\left.\left[\left(\mathrm{C}_{5} \mathrm{Me}_{5}\right) \mathrm{Rh}_{(\mathrm{OSMe}}\right)_{3}\right]^{2+}[31] \nu(\mathrm{SO})$ bei $926 \mathrm{~cm}^{-1}$ (in Nujol; O-Koordination) gefunden, für die analoge Iridiumverbindung in Lösung hingegen $\nu(\mathrm{SO}) 1128 \mathrm{~cm}^{-1}$ (S-Koordination). Die IR-Daten von 6 weisen auf Koordination des Liganden über das O-Atom hin, was aus sterischen Gründen plausibel erscheint (vgl. jedoch $\mathrm{CpMn}(\mathrm{CO})_{2}\left(\mathrm{Ph}_{2} \mathrm{SO}\right)\left(\nu(\mathrm{SO}) 1058 \mathrm{~cm}^{-1}\right)$ [28] und $\mathrm{ArCr}(\mathrm{CO})_{2}\left(\mathrm{Ph}_{2} \mathrm{SO}\right)$ [32] $(\mathrm{Ar}=$ Aryl; $\nu$ (SO) $\left.1090-1117 \mathrm{~cm}^{-1}\right)$.

\section{Umsetzungen mit Schwefeldioxid und Schwefelkohlenstoff}

Die Koordinationschemie von $\mathrm{SO}_{2}$ und $\mathrm{CS}_{2}$ ist vielfältig [33]. Sowohl einkernige Komplexe mit $\eta^{1}$ - [34] oder $\eta^{2}$-Koordination [35] als auch zwei- und mehrkernige Verbindungen mit $\eta^{1}-[36], \eta^{2}$ - [37] oder $\eta^{3}$ - [38] -Koordination sind bekannt. Hinweise auf die Bindungsart können auch aus den IR-Daten [33] und im Fall der $\mathrm{SO}_{2}$-Komplexe, aus der Reversibilität der Bindung und der Sauerstoffempfindlichkeit entnommen werden.

Die Verbindungen 7 und 8 konnten bisher wegen ihrer grossen Instabilität nicht eindeutig charakterisiert werden. Aus den IR-Spektren lassen sich jedoch folgende Schlüsse ziehen: wegen der hohen Lage der $\nu(\mathrm{CO})$-Banden müssen $\mathrm{SO}_{2}$ und $\mathrm{CS}_{2}$ als starke $\pi$-Akzeptoren wirken. Für $\mathrm{CS}_{2}$-Komplexe wird dies als typisch für $\eta^{2}$ Koordination angesehen [39]. Die Vielzahl der CO-Banden in 7 und 8 spricht für eine mindestens dimere, möglicherweise auch polymere Struktur. Für $\nu(E=X)$ werden in den als charakteristisch bezeichneten Bereichen keine intensiven Banden beobachtet. Die Banden bei 965 und $876 \mathrm{~cm}^{-1}$ sind allerdings vergleichbar denen im $\eta^{2}-\eta^{1}$-verbrückten Komplex $\left[\mathrm{Mo}(\mathrm{CO})_{2}\left(\mathrm{PPh}_{3}\right)_{2}(\mathrm{Py}) \mathrm{SO}_{2}\right]_{2}$ ( $\nu(\mathrm{SO}) 1043$ und 919 
$\mathrm{cm}^{-1}$ ) [38a] und in $\left[\mathrm{Cp}(\mathrm{CO})_{3} \mathrm{~W}-\mathrm{O}-\mathrm{S}\left(\mathrm{OSbF}_{5}\right) \mathrm{CH}_{3}\right]$ ( $v(\mathrm{SO}) 990,870$ und $845 \mathrm{~cm}^{-1}$ ) [40]. Bei der Pyrolyse von 7 konnte niemals $\mathrm{SO}_{2}$ festgestellt werden; offensichtlich ist das $\mathrm{SO}_{2}$ fester gebunden als $\mathrm{CO}$, das nachgewiesen werden konnte. Im Gegensatz dazu verlieren die $\mathrm{SO}_{2}-\mathrm{Komplexe}\left[\mathrm{CpFe}(\mathrm{CO})_{2} \mathrm{SO}_{2}\right]^{+} \mathrm{AsF}_{6}{ }^{-}$und $\left[\mathrm{CpCr}(\mathrm{NO})_{2}\left(\mathrm{SO}_{2}\right)\right]^{+}$ $\mathrm{AsF}_{6}{ }^{-}$und $\left[\mathrm{Mn}(\mathrm{CO})_{5} \mathrm{SO}_{2}\right]^{+} \mathrm{AsF}_{6}{ }^{-}$im Vakuum leicht $\mathrm{SO}_{2}$ unter Koordination des Anions [34a,41]. $\mathrm{SiF}_{4}$ und $\mathrm{CO}_{2}$ sind wohl als Folge von Hydrolysereaktionen (wegen der hohen Wasserempfindlichkeit sind 7 und 8 immer mit Spuren von $\mathrm{H}_{2} \mathrm{O}$ kontaminiert) am Anion und an koordiniertem $\mathrm{CO}$ zu betrachten. Die Bildung von $\left[\mathrm{Cp}(\mathrm{CO})_{3} \mathrm{MHM}(\mathrm{CO})_{3} \mathrm{Cp}\right]^{+}$unter Einfluss von Wasser lässt auf eine verbrückte Struktur von 7 und 8 schliessen, da die einkernigen Edukte 1 mit Wasser anders reagieren $[2,4,5]$.

\section{Reaktion mit Dithiocarbamat}

Mit Dithiocarbamatliganden $\mathrm{R}_{2} \mathrm{NCS}_{2}{ }^{-}$sind im wesentlichen drei Bindungstypen $\left(\eta^{1} ; \eta^{2} ; \mu-\eta^{1}, \eta^{1}\right)$ beschrieben worden, die alle im Cyclopentadienyl-wolfram-carbonyl-system beobachtet wurden [42]. Ein Komplex $\left[\mathrm{Cp}(\mathrm{CO})_{2} \mathrm{Fe}-\mathrm{SC}\left(\mathrm{NR}_{2}\right)\right.$ $\mathrm{S}-\mathrm{Fe}(\mathrm{CO})_{2} \mathrm{Cp}^{+}{ }^{+}$43] wurde kürzlich beschrieben. Bei der von uns durchgeführten Umsetzung von $\mathrm{CpM}(\mathrm{CO})_{3} \mathrm{FBF}_{3}\left(\mathrm{M}=\mathrm{Mo}\right.$, W) mit $\mathrm{NaS}_{2} \mathrm{CNHCH}_{2} \mathrm{Ph}$ entstehen keine Dithiocarbamato-Komplexe, sondern unter $\mathrm{H}^{+}$-Abspaltung die neutralen dimeren Dithiocarbimato-Komplexe 9:<smiles>O=C(COC(=O)NC(=O)OCc1ccccc1)OCc1ccccc1</smiles>

(9a: $M=$ Mo;

9b: $\mathbf{M}=\mathrm{W}$ )

Ein möglicherweise intermediär entstehender, zur oben genannten Eisenverbindung analoger kationischer Komplex wird anscheinend von überschüssigem $\mathrm{NaS}_{2} \mathrm{CNHCH}_{2} \mathrm{Ph}$, das als Base wirkt, deprotoniert. Die Verbindungen 9 sind im IR-Spektrum durch das Auftreten einer $\nu(C=N)$-Bande bei $1600 \mathrm{~cm}^{-1}$ charakterisiert. Verbrückende Dithiocarbimato-Komplexe wurden am Molybdän von Rakowsky-DuBois et al. beschrieben [44]. Vor allem mit Ni, Pd und $\mathrm{Pt}^{\mathrm{II}}$ sind zahlreiche Dithiocarbimato-Chelat-Komplexe bekannt [45].

\section{Experimenteller Teil}

Alle Reaktionen wurden in Schlenkrohren unter Argon als Schutzgas durchgeführt. Dichlormethan und Chloroform wurden nach üblichen Verfahren absolutiert und mit Argon gesättigt. Schwefelwasserstoff wurde aus $\mathrm{Na}_{2} \mathrm{~S}$ und Phosphorsäure hergestellt und über Molekularsieb $4 \AA$ getrocknet. Schwefelkohlenstoff (Merck, Darmstadt) wurde kurz vor Gebrauch unter Argon destilliert und sofort verwendet. Die übrigen Reagenzien waren handelsübliche Produkte und wurden ohne weitere Vorreinigung eingesetzt. Die Ausgangsverbindungen 1 wurden wie früher beschrieben oder analog dargestellt $[2,3,5]$.

Tricarbonyl(cyclopentadienyl)(dihydrogensulfid)molybdïn-tetrafluoroborat (2a) Auf $0.33 \mathrm{~g} \mathrm{CpMo}(\mathrm{CO})_{3} \mathrm{BF}_{4}(1.0 \mathrm{mmol})$ in $10 \mathrm{ml} \mathrm{CH}_{2} \mathrm{Cl}_{2}$ wird bei $-196^{\circ} \mathrm{C} 30$ 
min lang im Vakuum $\mathrm{H}_{2} \mathrm{~S}$ (aus einem auf $-70^{\circ} \mathrm{C}$ gekühlten Schlenkrohr, in das bei $-196^{\circ} \mathrm{C} \mathrm{H}_{2} \mathrm{~S}$ einkondensiert und dann auf $-70^{\circ} \mathrm{C}$ gebracht wurde) kondensiert. Es wird auf $-30^{\circ} \mathrm{C}$ erwärmt, wobei bald ein roter Niederschlag fält. Nach langsamem Temperaturanstieg auf $-15^{\circ} \mathrm{C}$ wird zentrifugiert und der isolierte Niederschlag 6.5 h bei -35 bis $0^{\circ} \mathrm{C}$ i. Hochvak. getrocknet. Ausb.: quantitativ.

Wegen der Instabilität von $\mathbf{2 a}$ konnten keine befriedigenden Analysen erhalten werden.

Tricarbonyl(cyclopentadienyl)(dihydrogensulfid)wolfram-hexafluoroarsenat ( $2 \boldsymbol{b}$ )

$0.46 \mathrm{~g} \mathrm{Ph}_{3} \mathrm{C}^{+} \mathrm{AsF}_{6}^{-}$werden in $10 \mathrm{ml} \mathrm{CHCl}_{3}$ suspendiert und bei $-40^{\circ} \mathrm{C}$ mit $\mathrm{CpW}(\mathrm{CO})_{3} \mathrm{H}$ versetzt, bis Farbumschlag nach violett erfolgt. Das Schlenkrohr wird evakuiert und dann mit $\mathrm{H}_{2} \mathrm{~S}$-Gas gefüllt. Nach $90 \mathrm{~min}$ wird abzentrifugiert, und die überstehende Lösung abdekantiert. Es wird zweimal mit je $5 \mathrm{ml} \mathrm{CH}_{2} \mathrm{Cl}_{2}$ und einmal mit $10 \mathrm{ml}$ Hexan bei $-50^{\circ} \mathrm{C}$ gewaschen und dann bei $-30^{\circ} \mathrm{C} 6 \mathrm{~h}$ i. Hockvak. getrocknet. (Gef.: $\mathrm{C}, 18.14 ; \mathrm{H}, 1.49 ; \mathrm{C}_{8} \mathrm{H}_{7} \mathrm{AsF}_{6} \mathrm{O}_{3} \mathrm{SW}$ ber.: $\mathrm{C}, 17.28 ; \mathrm{H}, 1.27 \%$. Molmasse 596.0).

Dicyclopentadienyl( $\mu$-hydrogensulfido)(hexacarbonyl)diwolframhexaftuoroantimonat (3)

$0.570 \mathrm{~g} \mathrm{CpW}(\mathrm{CO})_{3} \mathrm{FSbF}_{5}(1.0 \mathrm{~mol})$ in $10 \mathrm{ml} \mathrm{CHCl}{ }_{3}$ werden bei $-30^{\circ} \mathrm{C}$ mit einer Lösung von $0.148 \mathrm{~g} \mathrm{~N}^{\mathrm{n}} \mathrm{Bu}_{4}{ }^{+} \mathrm{SH}^{-}(0.54 \mathrm{mmol})$ in $5 \mathrm{ml} \mathrm{CHCl}$ behandelt. Nach 30 min wird abzentrifugiert und der Niederschlag 3 mal mit je $5 \mathrm{ml} \mathrm{CHCl}_{3}$ bei $-40^{\circ} \mathrm{C}$ gewaschen und dann $6 \mathrm{~h}$ im Ölpumpenvakuum getrocknet. (Gef.: C, 20.90; H, 1.71; $\mathrm{C}_{16} \mathrm{H}_{11} \mathrm{~F}_{6} \mathrm{O}_{6} \mathrm{SSbW}_{2}$ ber.: C, 20.60; H, 1.19\%; Molmasse 934.8).

\section{Deprotonierung von $\mathbf{2 b}$ und 3}

Bei $-50^{\circ} \mathrm{C}$ werden zu einer kleinen Probe von $2 \mathrm{~b} 10 \mathrm{ml} \mathrm{Et}{ }_{2} \mathrm{O}$ gegeben, wobei sich bald ein schwarzes Öl unter einer orangen Lösung bildet. Es wird weitergerührt, bis die Kühlbadtemperatur auf $-20^{\circ} \mathrm{C}$ gestiegen ist. Dann wird die Lösung abdekantiert und bei $-50^{\circ} \mathrm{C}$ zur Trockene eingeengt. Es bleibt ein gelbes Pulver zurück, das $2 \mathrm{~h}$ bei $-30^{\circ} \mathrm{C}$ i. Hochvak. getrocknet wird. IR: $\nu(\mathrm{CO}) 2036 \mathrm{~s}, 1938 \mathrm{vs}$, br (in $\mathrm{CH}_{2} \mathrm{Cl}_{2}$ ).

Eine kleine Probe von 3 wird bei $-60^{\circ} \mathrm{C}$ mit $20 \mathrm{ml} \mathrm{Et}{ }_{2} \mathrm{O}$ versetzt und langsam auf $-20^{\circ} \mathrm{C}$ erwärmt. Es wird eine Woche bei dieser Temperatur aufbewahrt. Dann wird abzentrifugiert und die schwachgelbe Lösung zur Trockene gebracht. Der Rückstand wird bei $-35^{\circ} \mathrm{C}$ mit $20 \mathrm{ml} \mathrm{Et}{ }_{2} \mathrm{O}$ und $47 \mu \mathrm{mol}$ Dicyclohexylethylamin in $\mathrm{Et}_{2} \mathrm{O}$ versetzt, wobei augenblickliche Grünfärbung auftritt. Abtrennen der Lösung und erneutes Versetzen mit etherischer Aminlösung werden zweimal wiederholt und die gesammelten Extrakte zur Trockene gebracht.

\section{Tricarbonylcyclopentadienyl(p-methylthiophenol)molybdìn-tetrafluoroborat (4a)}

$0.85 \mathrm{mmol} \mathrm{CpMo}(\mathrm{CO})_{3} \mathrm{FBF}_{3}$ werden in $10 \mathrm{ml} \mathrm{CH}_{2} \mathrm{Cl}_{2}$ bei $-30^{\circ} \mathrm{C}$ in situ dargestellt und mit $0.12 \mathrm{~g}(0.97 \mathrm{~mol}) p$-Methylthiophenol bei $-50^{\circ} \mathrm{C}$ versetzt. Es tritt sofort Reaktion ein. Das entstehende Öl, das mit Pentan vollständig ausgefällt wird, rührt man mehrere $h$ bei $-40^{\circ} \mathrm{C}$. Der nun ockerfarbene Niederschlag wird abgefrittet, $5 \mathrm{mal}$ mit je $10 \mathrm{ml}$ Pentan gewaschen und $1 \mathrm{~d} \mathrm{i}$. Hochvak. getrocknet: (Gef.: $\mathrm{C}, 38.66 ; \mathrm{H}, 2.07 ; \mathrm{C}_{15} \mathrm{H}_{13} \mathrm{BF}_{4} \mathrm{MoO}_{3} \mathrm{~S}$ ber.: $\mathrm{C}, 39.50 ; \mathrm{H}, 2.87 \%$. Molmasse 456.1).

Tricarbonyl $\left(\eta^{5}\right.$-indenyl)(p-methylthiophenol)wolfram-tetrafluoroborat (4b)

$0.79 \mathrm{mmol}\left(\eta^{5}-\mathrm{C}_{9} \mathrm{H}_{7}\right)(\mathrm{CO})_{3} \mathrm{WFBF}_{3}$ werden in $10 \mathrm{ml} \mathrm{CH}_{2} \mathrm{Cl}_{2}$ bei $-40^{\circ} \mathrm{C}$ in situ 
dargestellt und mit $0.097 \mathrm{~g}(0.78 \mathrm{mmol})$ para-Methylthiophenol versetzt. Nach etwa $2 \mathrm{~h}$ kann bei $-50^{\circ} \mathrm{C}$ ein gelber, flockiger Niederschlag isoliert werden, der fünfmal mit je $10 \mathrm{ml}$ Pentan gewaschen und $4 \mathrm{~h}$ i. Hochvak. getrocknet wird. (Gef.: C, 38.72; H, 3.08; $\mathrm{C}_{19} \mathrm{H}_{15} \mathrm{BF}_{4} \mathrm{O}_{3} \mathrm{SW}$ ber.: C, 38.42; $\mathrm{H}, 2.35 \%$. Molmasse 594.04).

Das entsprechende Hexafluoroarsenat-Salz 4c kann analog erhalten werden. (Gef.: C, 31.85; H, 2.10; $\mathrm{C}_{19} \mathrm{H}_{15} \mathrm{AsF}_{6} \mathrm{O}_{3} \mathrm{SW}$ ber.: C, 32.78; H, 2.17\%. Molmasse 696.2).

Dicarbonyl(cyclopentadienyl)(triphenylphosphit)(p-methylthiophenol)wolfram-tetrafluoroborat (4d)

$0.39 \mathrm{mmol} \mathrm{Cp}(\mathrm{CO})_{2} \mathrm{P}(\mathrm{OPh})_{3} \mathrm{WFBF}_{3}$ werden in $10 \mathrm{ml} \mathrm{CH}_{2} \mathrm{Cl}_{2}$ bei $-30^{\circ} \mathrm{C}$ dargestellt. Wenige Minuten nach Zugabe von $0.05 \mathrm{~g}(0.40 \mathrm{ml}) \quad p-\mathrm{CH}_{3} \mathrm{C}_{6} \mathrm{H}_{4} \mathrm{SH}$ wird mit $10 \mathrm{ml}$ Pentan ein rotes Öl gefällt. Die überstehende Lösung wird abpipettiert, der Rückstand durch Ausrühren mit $10 \mathrm{ml}$ Pentan bei $-50^{\circ} \mathrm{C}$ zur Kristallisation gebracht. Nach Abfritten und dreimaligem Waschen mit je $10 \mathrm{ml}$ Pentan wird $4 \mathrm{~h}$ i. Hochvak. getrocknet. (Gef.: $\mathrm{C}, 44.88 ; \mathrm{H}, 3.52 ; \mathrm{C}_{32} \mathrm{H}_{28} \mathrm{BF}_{4} \mathrm{O}_{5}$ PSW ber.: $\mathrm{C}, 46.52 ; \mathrm{H}$, 3.42\%. Molmasse 826.2).

\section{Tricarbonyl(cyclopentadienyl)(thiophenolato)wolfram}

$1.30 \mathrm{mmol}$ in situ dargestelltes $\mathrm{Cp}(\mathrm{CO})_{3} \mathrm{WFBF}_{3}$ werden bei $-30^{\circ} \mathrm{C}$ in $10 \mathrm{ml}$ $\mathrm{CH}_{2} \mathrm{Cl}_{2}$ mit $0.15 \mathrm{ml}(1.57 \mathrm{mmol})$ Thiophenol versetzt. Nach Temperaturanstieg auf $-10^{\circ} \mathrm{C}$ wird das Lösungsmittel i. Vak. abgezogen und das zurückbleibende Öl mit $10 \mathrm{ml} \mathrm{Et}{ }_{2} \mathrm{O}$ bei $-10^{\circ} \mathrm{C}$ gerührt, bis ein roter Niederschlag ausfällt. Man frittet ab, wäscht dreimal mit je $5 \mathrm{ml}$ Pentan und trocknet $4 \mathrm{~h}$ i. Hochvak. IR: $\nu(\mathrm{CO}): 2026 \mathrm{~s}$, 1911vs, br (in KBr). (Gef.: C, 37.70; H, 2.40; $\mathrm{C}_{14} \mathrm{H}_{10} \mathrm{O}_{3} \mathrm{SW}$ ber.: C, 38.03; H, 2.28\%. Molmasse 441.1).

\section{Tricarbonyl(cyclopentadienyl)(diphenylsulfid)molybdän-tetrafluoroborat (5a)}

Man stellt $0.82 \mathrm{mmol} \mathrm{Cp}(\mathrm{CO})_{3} \mathrm{MoFBF}_{3}$ in $10 \mathrm{ml} \mathrm{CH}_{2} \mathrm{Cl}_{2}$ bei $-30^{\circ} \mathrm{C}$ dar und versetzt bei $-50^{\circ} \mathrm{C}$ mit $0.15 \mathrm{ml}(0.94 \mathrm{mmol}) \mathrm{Ph}_{2} \mathrm{~S}$. Nach Erwärmung auf $-30^{\circ} \mathrm{C}$ wird mit $10 \mathrm{ml}$ Pentan ein gelbbrauner Niederschlag gefällt, der isoliert, $5 \mathrm{mal} \mathrm{mit} \mathrm{je}$ $10 \mathrm{ml}$ Pentan gewaschen und $3 \mathrm{~h}$ i. Hochvak. getrocknet wird.

Analog lassen sich auch die anderen Diphenylsulfidkomplexe 5 gewinnen, deren analytische Daten in Tab. 3 zusammengestellt sind.

\section{Tricarbonyl(cyclopentadienyl)(diphenylsulfoxid)molybdän-hexafluoroantimonat (6a)}

$0.60 \mathrm{mmol} \mathrm{Cp}(\mathrm{CO})_{3} \mathrm{MoFSbF}_{5}$ werden bei $-30^{\circ} \mathrm{C}$ in $10 \mathrm{ml} \mathrm{CH}_{2} \mathrm{Cl}_{2}$ dargestellt und bei $-50^{\circ} \mathrm{C}$ mit $0.16 \mathrm{~g}(0.79 \mathrm{mmol}) \mathrm{Ph}_{2} \mathrm{SO}$ versetzt. Man erwärmt im Laufe von $3 \mathrm{~h}$ auf $-20^{\circ} \mathrm{C}$ und fällt mit $10 \mathrm{ml}$ Pentan einen kirschroten Niederschlag aus, der abgefrittet, 4 mal mit je $10 \mathrm{ml}$ Pentan gewaschen und $5 \mathrm{~h}$ i. Hochvak. getrocknet wird. (Gef.: C, 34.01; H, 1.86; $\mathrm{C}_{20} \mathrm{H}_{15} \mathrm{~F}_{6} \mathrm{MoO}_{4}$ Sb ber.: $\mathrm{C}, 35.17 ; \mathrm{H}, 2.21 \%$. Molmasse 683.1).

\section{Tricarbonyl(cyclopentadienyl)(diphenylsulfoxid)wolfram-hexafluoroantimonat (6b)}

$0.63 \mathrm{mmol}$ von in situ dargestelltem $\mathrm{Cp}(\mathrm{CO})_{3} \mathrm{WFSbF}_{5}$ werden $24 \mathrm{~h}$ mit $0.16 \mathrm{~g}$ ( $0.79 \mathrm{mmol}) \mathrm{Ph}_{2} \mathrm{SO}$ bei $-30^{\circ} \mathrm{C}$ gerührt. Mit $10 \mathrm{ml}$ Pentan wird ein braunrotes Öl gefällt, von dem die überstehende Lösung abpipettiert wird. Das Öl wird bei RT mit $15 \mathrm{ml} \mathrm{Et}{ }_{2} \mathrm{O}$ gerührt, bis ein karminrotes Pulver entsteht, das isoliert, fünfmal mit je 
TABELLE 3

ANALYTISCHE DATEN VON 5

\begin{tabular}{llcc}
\hline & $\mathrm{M}$ & \multicolumn{3}{c}{ Gef. (ber.) (\%) } \\
\cline { 3 - 4 } & & $\mathrm{C}$ & $\mathrm{H}$ \\
\hline $\mathrm{C}_{20} \mathrm{H}_{15} \mathrm{BF}_{4} \mathrm{MoO}_{3} \mathrm{~S}$ & 518.1 & 46.01 & 3.16 \\
$(5 \mathrm{5a})$ & & $(46.36)$ & $(2.92)$ \\
$\mathrm{C}_{20} \mathrm{H}_{15} \mathrm{BF}_{4} \mathrm{O}_{3} \mathrm{SW}$ & 606.0 & 38.57 & 2.82 \\
$(\mathbf{5 b})$ & & $(39.64)$ & $(2.49)$ \\
$\mathrm{C}_{20} \mathrm{H}_{15} \mathrm{~F}_{6} \mathrm{O}_{3} \mathrm{SSbW}$ & 755.0 & 31.12 & 1.87 \\
$(5 \mathbf{5 c})$ & & $(31.82)$ & $(2.00)$ \\
$\mathrm{C}_{24} \mathrm{H}_{17} \mathrm{AsF}_{6} \mathrm{O}_{3} \mathrm{SW}$ & 758.2 & 37.07 & 2.11 \\
$(\mathbf{5 d})$ & & $(38.02)$ & $(2.26)$ \\
\hline
\end{tabular}

$10 \mathrm{ml}$ Pentan gewaschen und einen Tag i. Hochvak. getrocknet wird. (Gef.: C, 28.24; $\mathrm{H}, 2.00 ; \mathrm{C}_{20} \mathrm{H}_{15} \mathrm{~F}_{6} \mathrm{O}_{4} \mathrm{SSbW}$ ber.: $\mathrm{C}, 31.16 ; \mathrm{H}, 1.96 \%$. Molmasse 771.0).

Reaktionen von $\mathrm{Cp}(\mathrm{CO})_{3} \mathrm{WFEF}_{5}(\mathrm{E}=\mathrm{P}, \mathrm{Sb})$ mit $\mathrm{SO}_{2}$

(a) Etwa $2 \mathrm{ml} \mathrm{SO}{ }_{2}$ werden in ein Schlenkrohr bei $-50^{\circ} \mathrm{C}$ einkondensiert. Bei Zugabe von etwas $\mathrm{Cp}(\mathrm{CO})_{3} \mathrm{WFPF}_{5}$ entsteht sofort eine tiefrote Lösung. Nach 15 min wird das $\mathrm{SO}_{2}$ bei $-50^{\circ} \mathrm{C}$ i. Vak. abgezogen und der Rückstand bei langsam auf $0^{\circ} \mathrm{C}$ steigender Temperatur getrocknet.

Das so erhaltene Produkt wird mehrere Tage auf $+85^{\circ} \mathrm{C}$ i. Vak. erhitzt, die entstehenden gasförmigen Produkte fängt man in einer Gasküvette auf. IRspektroskopisch können $\mathrm{SiF}_{4}\left(1028 \mathrm{~cm}^{-1}\right) \mathrm{CO}\left(2140 \mathrm{~cm}^{-1}\right)$ und $\mathrm{CO}_{2}\left(2330 \mathrm{~cm}^{-1}\right)$ identifiziert werden.

(b) Festes $\mathrm{CpW}(\mathrm{CO})_{3} \mathrm{FSbF}_{5}$ wird in einem Schlenkrohr auf $-30^{\circ} \mathrm{C}$ gekühlt. Es wird evakuiert und mit $\mathrm{SO}_{2}$-Gas gefüllt. Nach mehreren Stunden wird das purpurfarbene Produkt i. Hochvak. bei Temperaturen unter $-10^{\circ} \mathrm{C}$ getrocknet. (Analyse: C, 23.30; $\mathrm{H}, 1.65 \%)$. IR ( $\mathrm{cm}^{-1}$, in Nujol): $2173 \mathrm{~m}, 2133 \mathrm{~s}, 2085 \mathrm{sh}, 2057 \mathrm{vs}, 1952 \mathrm{vs}$, br ( $\left.\nu(\mathrm{CO})\right)$ : $1108 \mathrm{w}, 1067 \mathrm{w}, 965 \mathrm{~s} ; 876 \mathrm{~m}(\nu(\mathrm{SO}))$.

Reaktion von $\mathrm{CP}(\mathrm{CO})_{3} \mathrm{MoFBF}_{3}$ mit $\mathrm{CS}_{2}$

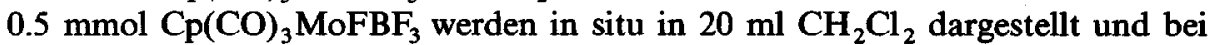
$-40^{\circ} \mathrm{C}$ mit $2.5 \mathrm{mmol} \mathrm{CS}{ }_{2}$ versetzt. Nach $1 \mathrm{~min}$ fällt ein Niederschlag aus, der nach 3 $\mathrm{h}$, wobei eine Temperatur von $-10^{\circ} \mathrm{C}$ erreicht ist, abgefrittet und bei $25^{\circ} \mathrm{C} 3 \mathrm{~h} \mathrm{i}$. Hochvak. getrocknet wird. IR ( $\mathrm{cm}^{-1}$, in Nujol): 2162w, 2143m, 2123w, 2073vs, 2048vs, 2003sh, 1968vs, br $(\nu(\mathrm{CO}))$ : $1124(\nu(\mathrm{CS})) .{ }^{1} \mathrm{H}-\mathrm{NMR}$ (in Aceton- $\left.d_{6}\right): \delta 7.4 \mathrm{~m}$, $6.75 \mathrm{~s}, \quad 6.55 \mathrm{~s}, 6.46 \mathrm{~s} \quad\left(\left[\mathrm{CpMo}(\mathrm{CO})_{4}\right]^{+}\right), 6.17 \quad\left(\left[\mathrm{CpMo}(\mathrm{CO})_{3}\left(\mathrm{H}_{2} \mathrm{O}\right)\right]^{+}\right.$und $\left.\left[\mathrm{Cp}_{2} \mathrm{Mo}_{2}(\mathrm{CO})_{6} \mathrm{H}\right]^{+}\right), 6.11 \mathrm{~s},-20.4 \mathrm{~s}\left(\left[\mathrm{Cp}_{2} \mathrm{Mo}_{2}(\mathrm{CO})_{6} \mathrm{H}\right]^{+}\right)$(in ppm).

Reaktion von $\mathrm{CpW}(\mathrm{CO})_{3} \mathrm{FAsF}_{5}$ mit $\mathrm{CS}_{2}$

Etwa $0.30 \mathrm{mmol} \mathrm{Cp}(\mathrm{CO})_{3} \mathrm{WFAsF}_{5}$ werden in $10 \mathrm{ml} \mathrm{CH}_{2} \mathrm{Cl}_{2}$ bei $-30^{\circ} \mathrm{C}$ mit 0.02 $\mathrm{ml} \mathrm{CS} 2(0.33 \mathrm{mmol})$ versetzt. Der nach 30 min ausfallende braune Niederschlag wird abzentrifugiert und bei $-20^{\circ} \mathrm{C} 6 \mathrm{~h}$ i. Hochvak. getrocknet. (Analyse: C, 17.71; $\mathrm{H}$, $1.46 \%)$. IR ( $\mathrm{cm}^{-1}$, in Nujol): $2155 \mathrm{~m}, 2115 \mathrm{~s}, 2050 \mathrm{vs}, 1978 \mathrm{vs,} 1950 \mathrm{vs}$ ( $\nu(\mathrm{CO})$ ); $1145 \mathrm{~m}$ $(\nu(\mathrm{CS}))$.

$\mu$-(N-Benzyldithiocarbimato)bis(tricarbonylcyclopentadienylmolybdian) (9a)

Etwa $0.9 \mathrm{mmol}$ von in situ dargestelltem $\mathrm{Cp}(\mathrm{CO})_{3} \mathrm{MoFBF}_{3}$ werden bei $-30^{\circ} \mathrm{C}$ in 
$10 \mathrm{ml} \mathrm{CH}_{2} \mathrm{Cl}_{2}$ mit $1.25 \mathrm{mmol} \mathrm{NaS} \mathrm{CNHCH}_{2} \mathrm{C}_{6} \mathrm{H}_{5}$ versetzt. Es tritt fast augenblicklich Verfärbung nach rot auf und ein Niederschlag fällt aus. Nach $18 \mathrm{~h}$ sind $10^{\circ} \mathrm{C}$ erreicht und es wird abgefrittet. Das Filtrat wird zur Trockene eingeengt und mit 10 $\mathrm{ml} \mathrm{Et}{ }_{2} \mathrm{O}$ gerührt. Der entstandene pulverige Niederschlag wird isoliert und $4 \mathrm{~h} \mathrm{im}$ Ölpumpenvakuum getrocknet. (Gef.: C, 43.02; $\mathrm{H}, 2.96 ; \mathrm{N}, 2.07 ; \mathrm{C}_{24} \mathrm{H}_{17} \mathrm{Mo}_{2} \mathrm{NO}_{6} \mathrm{~S}_{2}$ ber. C, 42.9; H, 2.55; N, 2.09\%. Molmasse 671.4).

$\mu$-( $N$-Benzyldithiocarbimato)bis(tricarbonylcyclopentadienylwolfram)(9b)

In eine Lösung von etwa $0.9 \mathrm{mmol} \mathrm{Cp}(\mathrm{CO})_{3} \mathrm{WFBF}_{3}$ in $10 \mathrm{ml} \mathrm{CH}_{2} \mathrm{Cl}_{2}$ werden bei $-27^{\circ} \mathrm{C} 1.0 \mathrm{mmol} \mathrm{NaS}_{2} \mathrm{CNHCH}_{2} \mathrm{C}_{6} \mathrm{H}_{5}$ gegeben, wobei sofortige Rotfärbung auftritt. Nach 4 h sind $-10^{\circ} \mathrm{C}$ erreicht und es wird abgefrittet. Der grauschwarze Rückstand wird mit dreimal $10 \mathrm{ml} \mathrm{Et}_{2} \mathrm{O}$ gewaschen, die vereinigten Filtrate werden auf Trockeneis gestellt. Nach $18 \mathrm{~h}$ wird der entstandene Niederschlag isoliert und $1 \mathrm{~h}$ i. Hochvak. bei RT getrocknet. (Gef.: C, 34.69; H, 2.55; N, 1.67; $\mathrm{C}_{24} \mathrm{H}_{17} \mathrm{NO}_{6} \mathrm{~S}_{2} \mathrm{~W}_{2}$ ber.: C, 34.00; H, 2.02; N, 1.65\%. Molmasse 847.2).

\section{Dank}

Der Deutschen Forschungsgemeinschaft und dem Fonds der Chemischen Industrie gilt unser besonderer Dank für die Förderung dieser Arbeiten. Frl. D. Motz danken wir für wertvolle experimentelle Mitarbeit.

\section{Literatur}

1 K. Sünkel, G. Urban und W. Beck, J. Organomet. Chem., 290 (1985) 231.

2 W. Beck und K. Schloter, Z. Naturforsch, B, 33 (1978) 1214.

3 K. Sünkel, H. Ernst und W. Beck, Z. Naturforsch. B, 36 (1981) 474.

4 K. Schloter, U. Nagel und W. Beck, Chem. Ber., 113 (1980) 3775.

5 K. Sünkel, G. Urban und W. Beck, J. Organomet. Chem., im Druck.

6 K. Schloter und W. Beck, Z. Naturforsch. B, 35 (1980) 985.

7 (a) W. Danzer, W.P. Fehlhammer, A.T. Liu, G. Thiel und W. Beck, Chem. Ber., 115 (1982) 1682; (b) vgl. auch A. Shaver, J. Hartgerink, R.D. Lal, P. Bird und N. Ansari, Organometallics, 2 (1983) 938.

8 M. Herberhold, W. Jellen und H.H. Murray, J. Organomet. Chem., 270 (1984) 65.

9 R. Havlin und G.R. Knox, Z. Naturforsch. B, 21 (1966) 1108.

10 (a) D. Coucouvanis, Progr. Inorg. Chem., 11 (1970) 233; (b) E.J. Kupchik und P.J. Calabretta, Inorg. Chem., 4 (1965) 973.

11 C.G. Kuehn und H. Taube, J. Amer. Chem. Soc., 98 (1976) 689.

12 (a) M. Herberhold und G. Süss, J. Chem. Kes., (M) (1977) 2720; (b) M. Herberhold und G. Süss, Angew. Chem., 88 (1976) 375.

13 P.J. Harris, S.A.R. Knox, R.J. McKinney und F.G.A. Stone, J. Chem. Soc., Dalton Trans., (1978) 1009.

14 K. Raab und W. Beck, Chem. Ber., im Druck.

15 A.M. Mueting, P. Boyle und L.H. Pignolet, Inorg. Chem., 23 (1984) 44.

16 K. Osakada, T. Yamamoto und A. Yamamoto, Inorg. Chim. Acta, 90 (1984) L5 und dort zitierte Literatur.

17 R.G.W. Gingerich und R.J. Angelici, J. Amer. Chem. Soc., 101 (1979) 5604.

18 M. Höfler und A. Baitz, Chem. Ber., 109 (1976) 3147.

19 V. Küllmer und H. Vahrenkamp, Chem. Ber., 109 (1976) 1560.

20 T.J. Greenhough, B.W.S. Kolthammer, P. Legzdins und J. Trotter, Inorg. Chem., 18 (1979) 3543.

21 M.D. Curtis und W.M. Butler, J. Chem. Soc. Chem. Commun., (1980) 998.

22 Vgl. z.B. (a) D. Mohr, H. Wienand und M.L. Ziegler, Z. Naturforsch. B, 31 (1976) 66; (b) P.M. Treichel und G.R. Wilkes, Inorg. Chem., 5 (1966) 1182; (c) W. Ehrl und H. Vahrenkamp, Chem. Ber., 105 (1972) 1471; (d) D.D. Watkins Jr. und T.A. George, J. Organomet. Chem., 102 (1975) 71; (e) I.B. 
Benson, S.D. Killops, S.A.R. Knox und A.J. Welch, J. Chem. Soc., Chem. Commun., (1980) 1137; (f) J.C.T.R. Burckett-St. Laurent, M.R. Caira, R.B. English, R.J. Haines und L.R. Nassimbeni, J. Chem. Soc., Dalton Trans., (1977) 1077.

23 P.M. Treichel und L.D. Rosenhein, Inorg. Chem., 20 (1981) 942.

24 S.G. Murray und F.R. Hartley, Chem. Rev., 81 (1981) 365.

25 M.J.H. Russell, C. White, A. Yates und P.M. Maitlis, J. Chem. Soc., Dalton Trans., (1978) 849.

26 W.E. Williams und F.J. Lalor, J. Chem. Soc., Dalton Trans., (1973) 1329.

27 H. Werner und B. Juthani, Z. Anorg. Allg. Chem., 473 (1981) 107.

28 W. Strohmeier und J.F. Guttenberger, Chem. Ber., 97 (1964) 1871.

29 Vgl. z.B. J.A. Davies und F.R. Hartley, Chem. Rev., 81 (1981) 79.

30 W.L. Reynolds, Progr. Inorg. Chem., 12 (1970) 1.

31 C. White, S.J. Thompson und P.M. Maitlis, J. Chem. Soc., Dalton Trans., (1977) 1654.

32 W. Strohmeier, G. Popp und J.F. Guttenberger, Chem. Ber., 99 (1966) 165.

33 Reviews: (a) D.M.P. Mingos, Trans. Met. Chem., 3 (1978) 1; (b) G.J. Kubas, Inorg. Chem., 18 (1979) 182; (c) P.V. Yaneff, Coord. Chem. Rev., 23 (1977) 183; (d) I.S. Butler und A.E. Fenster, J. Organomet. Chem., 66 (1974) 161.

34 (a) mit $\mathrm{SO}_{2}$ : z.B. G. Hartmann, R. Froböse, R. Mews und G.M. Sheldrick, Z. Naturforsch. B, 37 (1982) 1234; G.A. Gott, J. Fawcett, C.A. McAuliffe und D.R. Russell, J. Chem. Soc., Chem. Commun., (1984) 1283; (b) mit CS CS $_{\text {L }}$. Ruiz-Ramirez, T.A. Stephenson und E.S. Switkes, J. Chem. Soc., Dalton Trans., (1973) 1770.

35 (a) mit $\mathrm{SO}_{2}$ : R.R. Ryan und D.C. Moody, J. Chem. Soc., Chem. Commun., (1976) 503; (b) mit $\mathrm{CS}_{2}$ : M. Herberhold, M. Süss-Fink und C.G. Kreiter, Angew. Chem., 89 (1977) 191.

36 mit $\mathrm{SO}_{2}$ : M. Angoletta, P.L. Bellon, M. Manassero und M. Sansoni, J. Organomet. Chem., 81 (1974) $\mathrm{C} 40$.

37 mit CS$_{2}$ : J.E. Ellis, R.W. Fennell und E.A. Flom, Inorg. Chem., 15 (1976) 2031.

38 (a) mit $\mathrm{SO}_{2}$ : G.D. Jarvinen, G.J. Kubas, R.R. Ryan, J. Chem. Soc., Chem. Commun., (1981) 305; (b) mit CS 2 : J.M. Lisy, E.D. Dobrzynski, R.J. Angelici und J. Clardy, J. Amer. Chem. Soc., 97 (1975) 656; H. Stolzenberg, W.P. Fehlhammer, P. Dixneuf, J. Organomet. Chem., 246 (1983) 105; C. Bianchini, C. Mealli, A. Meli, A. Orlandini und L. Sacconi, Inorg. Chem., 19 (1980) 2968.

39 M. Herberhold und M. Süss-Fink, Angew. Chem., 89 (1977) 192.

40 R.G. Severson und A. Wojcicki, J. Amer. Chem. Soc., 101 (1979) 877.

41 R. Mews, Angew. Chem., 87 (1975) 669.

42 H.B. Abrahamson, M.L. Freeman, M.B. Hossain und D. van der Helm, Inorg. Chem., 23 (1984) 2286.

43 D. Catheline und D. Astruc, Organometallics, 3 (1984) 1094.

44 D.J. Miller und M. Rakowsky-DuBois, J. Amer. Chem. Soc., 102 (1980) 4925.

45 (a) R. Schierl, U. Nagel und W. Bock, Z. Naturforsch. B, 39 (1984) 649; (b) C.A. Tsipis, I.J. Meleziadis, D.P. Kessissoglou und G.A. Katsoulos, Inorg. Chim. Acta, 90 (1984) L19 und dort zitierte Literatur. 